\title{
The Evolution of the Mass-Metallicity Relation in Seyferts
}

\section{N. Vale Asari ${ }^{1,2}$, G. Stasińska ${ }^{2}$, R. Cid Fernandes ${ }^{1}$, J. M. Gomes ${ }^{1,3}$, M. Schlickmann ${ }^{1}$, A. Mateus ${ }^{4}$, and W. Schoenell ${ }^{1}$}

${ }^{1}$ Dpto. de Física - CFM - Universidade Federal de Santa Catarina, Florianópolis, SC, Brazil

${ }^{2}$ LUTH, Observatoire de Paris, CNRS, Université Paris Diderot; Place Jules Janssen 92190

Meudon, France

${ }^{3}$ GEPI, Observatoire de Paris, CNRS, Université Paris Diderot; Place Jules Janssen 92190

Meudon, France

${ }^{4}$ IAG, Universidade de São Paulo, São Paulo, SP, Brazil

Keywords. galaxies: evolution, galaxies: statistics, galaxies: stellar content.

Various studies have shown that there is an empirical relation between the nebular metallicity of a galaxy and its stellar mass. Until now, most studies of the mass-metallicity relation $(M-$ $Z$ ) have focused on the abundances of the interstellar medium as measured by emission-line features. This technique thus excludes galaxies with AGN from the working samples, due to the difficulty to measure the nebular abundances when emission-lines are powered both by stars and AGN. With our synthesis code STARLIGHT, we are able to recover the stellar metallicities of galaxies from the Sloan Digital Sky Survey (SDSS). Therefore, although we still cannot measure the nebular metallicity in AGN hosts, we know their present-day stellar metallicities. Moreover, because we measure the metallicity of stellar populations of different ages in a galaxy, we are also able also recover the history of its chemical enrichment.

In this work, we show the present-day $M-Z$ relation and its evolution for Seyfert galaxies in the SDSS. We find that the chemical enrichment and the star formation of Seyferts is much faster than for a star-forming galaxy of the same present-day mass. We show that, for presentday stellar masses above $10^{10} M_{\odot}$, SDSS galaxies can be explained well with a simple closed-box model. We also find that the evolution of Seyferts is more similar to galaxies that have already stopped forming stars than to star forming galaxies.

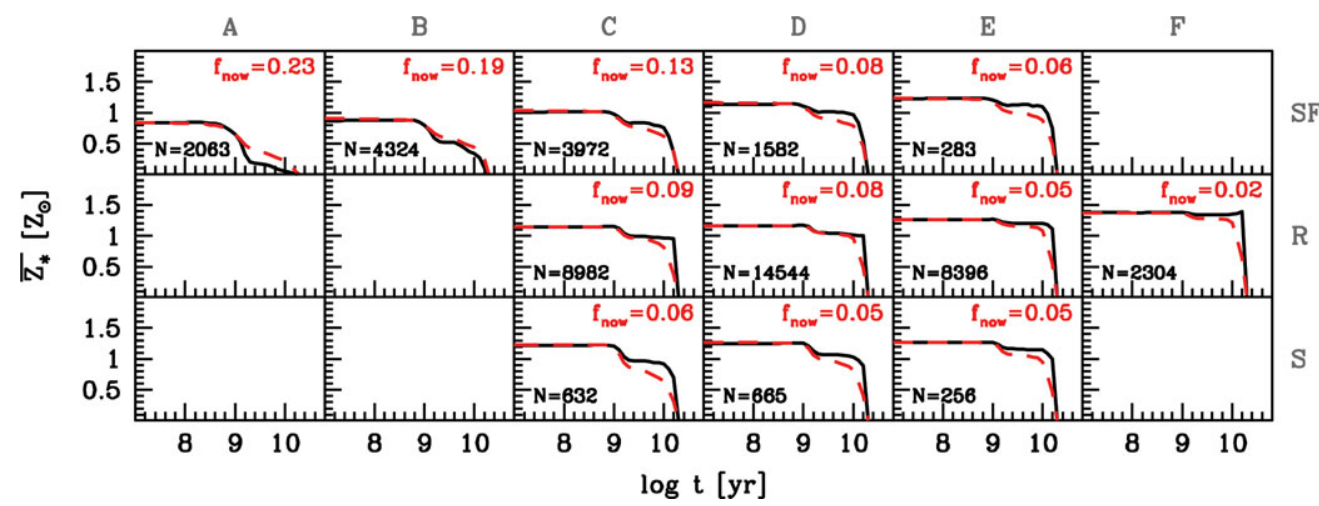

Figure 1. Median of the evolution of stellar metallicity as found by STARLIGHT (solid lines) and as obtained with the simple closed-box model (dashed lines). The number of objects $(N)$ in each panel is on the bottom and the present-day gas mass fraction $\left(f_{\text {now }}\right)$ is on the top. Our sample has been divided in mass-bins $\mathrm{A}-\mathrm{F}$ and by galaxy type: star-forming $(\mathrm{SF})$, retired/passive $(\mathrm{R})$ and Seyfert (S). A full version of this study is presented by Vale Asari et al. (2009).

\section{Reference}

Vale Asari, N., Stasińska, G., Cid Fernandes, R., Gomes, J. M., Schlickmann, M., Mateus, A., \& Schoenell, W. 2009, MNRAS, 396, L71 